\title{
Towards Meta-Adaptation Support with Reusable and Composable Adaptation Components
}

\author{
Ning Gui \\ Zhejiang Sci-Tech University, China and PATS group, \\ University of Antwerp, Belgium and IBBT, Belgium \\ e-mail: ninggui@gmail.com
}

\author{
Vincenzo De Florio \\ PATS group, Dept. of Mathematics and Computer Science \\ UA and IBBT, Belgium \\ e-mail: vincenzo.deflorio@ua.ac.be
}

\begin{abstract}
Software systems today are increasingly used in changing environments and expected to adapt with variable adaptation concerns. This requirement demands a systematic approach to efficiently construct system global adaptation behaviour according to the dynamic adaptation requirements. This paper presents Transformer - a framework for adaptation behaviour composition support based on reusable and composable adaptation components. Rather than using one adaptation module for all possible contexts, Transformer constructs system global adaptation behaviour by contextually fusing adaptation plans from multiple adaptation components. Explicit conflict resolution is provided to handle possible conflicts raised in the fusion process. In addition to the description of the Transformer framework, this paper also presents its implementation and its application to a video conferencing system. Qualitative analysis and simulation results show that our framework exhibits significant advantage over traditional approaches in light of flexibility and reusability of the adaptation components with little performance overhead.
\end{abstract}

Keywords - self-adaptive software; adaptation composition; conflict resolution; meta-adaptation.

\section{INTRODUCTION}

With the fast development of mobile and ubiquitous computing, software systems are more and more used in changing environments. As such, they are increasingly expected to dynamically self-adapt to accommodate for resource variability, changing user needs, and system faults - in most cases, with different and changing combinations of these factors. This means that system adaptation modules should be designed to respect changing and multiple adaptation concerns - for instance, achieving both selfoptimization and self-healing behaviours at the same time.

While runtime adaptation with multiple concerns is desirable, the realization of this feature is challenging, especially when systems work in changing contexts. In different contexts, systems might have different sets of adaptation concerns. This dynamicity makes the traditional static solution - a single global adaptation module covering all situations - rather unpractical. As pointed out by Oreizy [3], for a changing environment, not only the adaptive software needs to be changed to reflect new context requirements - the system adaptation behaviour itself should also evolve to reflect these changes. Hillman and Warren [5] denote this process as "meta-adaptation."
This paper provides a comprehensive description of Transformer, an architecture-based adaptation framework that offers compositional adaptation support for building self-adaptive software. In this framework, adaptation strategies are consolidated into well-defined adaptation controllers - denoted as Composable Adaptation Planners (CAPs) - to facilitate the composition of multiple adaptation behaviours (adaptation composition). The most important feature of Transformer is that system global adaptation behaviour is constructed with multiple CAPs during runtime, according to the context to date, just as components are assembled to build applications. As CAPs might generate conflicting adaptation plans, Transformer provides a systematic coordination mechanism to achieve effective and correct composition. It also allows prototyping, testing, evaluation and injection of new adaptation behaviours for component-based adaptable software.

A major goal of this work is to present a general adaptation framework that can use future unspecified adaptation components instead of a domain-specific solution that can only be used for particular application-specific environments or has to be re-implemented each time when context requirements change. In order to achieve this objective, the design principles of adaptation components are identified. While other frameworks have been proposed with similar goals (e.g., Rainbow [8], CARISMA [9], Cholla [10]), to the best of our knowledge none of them focuses on the conflict detection and resolution to the extent that is done here. This work is based on our previous ACCADA framework [11] with extensions for adaptation composition and conflict resolution mechanisms.

\section{MOTIVATIONAL EXAMPLE \& CHALLENGES}

In this section we firstly describe a motivational example to demonstrate the need for meta-adaptation support. Then the challenges in realizing this example are identified.

\section{A. Motivational Example}

Let us suppose that user John needs to run a video conferencing system on a mobile device. As shown in the lower part of Fig. 1, this application consists of three major sub-systems. The Jabber subsystem is used for messaging and presentation transport; the local media capture subsystem captures local video/audio data and sends them to a remote stream server; the remote media player subsystem is used to play back media data from remote peers. 


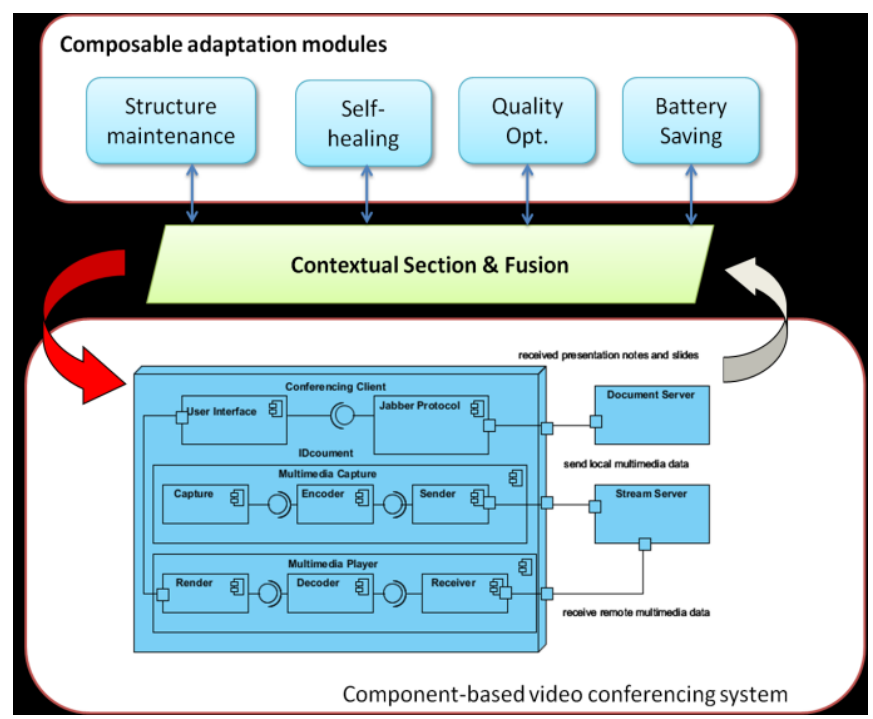

Figure 1. Contextual Adaptation Behaviour Composition.

This video conferencing system is expected to adapt its behaviour to meet the changing requirements of the system status and/or John's preferences. Even this very simple scenario, when adapting within a changing environment, calls for complex treatment, which can be more easily described by a few scenarios:

Quality Optimization: John is actively participating in the conference and would like to experience the best quality in this application. Adaptation logics can be: always adjust video Encoder's configuration with the best possible quality that the current system allows. In order to address this adaptation concern, an adaptation module - adaptation module for quality optimization - needs to be implemented.

Battery Saving: When the remaining battery is below a certain threshold or John is not actively involved in the meeting, he would prefer to reduce the video encoding quality or even disable the local video encoder to save energy when the battery becomes critically low. Consequently we need an adaptation module - adaptation module for battery saving - to implement such strategy for this context.

Balanced: With the battery level shifting from high to low, John would like to have a balanced configuration to consider both the above adaptation concerns and reflect this gradual context shift.

In all the above cases, John would like to have "selfhealing" capabilities for the enabled applications in order to deal with possible application crashes.

What above are just three cases out of many possible situations that John might experience. For each new scenario, a new adaptation module is needed to represent a new set of adaptation requirements. However, developing an adaptation module is not a trivial endeavour. For instance, in the "Quality Optimization" scenario, an adaptation module needs to handle adaptation concerns from at least three different aspects: 1) application structural maintenance for building and maintaining application structure during runtime - this function is necessary to support dynamic encoder selection and is an important feature in supporting compositional adaptation [12]; 2) adaptation logics for optimized video quality; 3 ) self-healing adaptation logics to tolerate possible application crashes [13].

In traditional approaches, a monolithic adaptation module is normally used to statically integrate different adaptation logics. When the context changes, for instance, from "Quality Optimization" to "Battery Saving", the adaptation module will not work correctly despite these two contexts have many adaptation requirements in common. We argue that a better solution to address such problem is to divide the adaptation modules into small adaptation building blocks representing particular adaptation goals matching well-defined "scenarios". Each block assumes that some contextual hypotheses are valid and focuses on one particular adaptation concern. These adaptation blocks can be used to construct more complex adaptation modules and could (possibly) be reused across multiple contexts. In so doing, the system global adaptation module is built by selecting a set of such "blocks" and "fusing" them. Compared to the one-size-fits-all (monolithic) approach, this solution simplifies the adaptation module development modular design allows reuse of existing adaptation modules. As depicted in Fig. 1, the self-healing module and structural maintenance module can be (re)used across all three above scenarios.

\section{B. Challenges}

Although adaptation behaviour composition shows interesting features in achieving adaptation reusability and supporting changing adaptation concerns, however, in order to achieve such features, several challenges remain to be tackled.

The first challenge is to enhance the reusability and composability of adaptation modules. From the design perspective, most adaptation frameworks $[1,4,14]$ provide no clear design principles to facilitate the composition of multiple adaptation modules. Lack of those guidelines makes adaptation components very hard, if not impossible, for those modules to be composed with each other.

The second challenge is allowing meta-adaptation. Due to the changing environments, adaptation modules must evolve to match their new requirements. For instance, in the motivational example, when the context migrates from Quality Optimization to Battery Saving, adaptation framework should allow the shift of employed adaptation modules and guarantee that the most appropriate set of adaptation logics are used for adaptation. Many existing adaptive systems allow their adaptation modules to evolve through AI based learning algorithm, such as the decision tree-based learning algorithm used in [15]. However, they normally need considerable training data to acquire new adaptation knowledge. Thus, such algorithms are not capable to deal with fast changing and unexpected contexts. 
The third challenge is to provide conflict detection and resolution support. This becomes a natural requirement for system design when system global adaptation behaviour is constructed by merging possibly conflicting adaptation modules. However, often solutions do not provide any conflict resolution process, e.g. AAOP [6] and the opportunistic adaptation integration [7]. Others only provide static integration mechanisms $[1,10,16]$ that are tailored for particular application domains. In these approaches, conflict resolution logics normally are intertwined with adaptation logics in the form of binary code. This strong coupling makes those fusion logics very hard to manage or revise. Some researchers did identify the importance of conflict detection and resolution [8, 9]. Utility functions are normally used to resolve those conflicts. However, as pointed out by the Research Roadmap of self-adaptive systems [17], utility functions are very challenging to define and need to be redefined as environments change.

In order to deal with these challenges, it is argued, the composable adaptation component, meta-adaptation and conflict resolution have to be elicited from other adaptation processes and explicitly supported by the adaptation framework. This separation is the cornerstone of the Transformer framework as it provides a clearer view to the adaptation processes by allowing modular design of adaptive systems.

\section{TRANSFORMER ADAPTATION FRAMEWORK}

Following the analysis of the design issues, this section introduces the Transformer framework from different viewpoints. We begin by explaining the key modules of our architecture as well as their interactions. Then we zoom in on each key module in the following sections.

\section{A. Framework Architecture Overview}

As shown in Fig. 2, the Transformer framework takes architecture-based adaptation and provides an external adaptation loop with respect to managed adaptive software. It contains six types of modules - Event Monitor, Adaptation Actuator, System Basic Run-time (SBR), Model Fusion, CAP, CAP Selector and Validation modules. These modules realize an external control loop that is separated from underlying applications constructed by adaptable components ${ }^{1}$.

- System Basic Run-time (SBR). The SBR provides services necessary to support adaptive component management and adaptation process with multiple CAPs. It 1) installs, discovers, executes and uninstalls components, as well as manages the component instance registry; 2) provides the service registry support and monitors service changes; 3 ) manages an updated global image. This instance contains the meta-objects for all installed components. A component meta-object contains component instance-related information such as component states, properties as well as the dependences

\footnotetext{
${ }^{1}$ In what follows we shall refer to adaptable components as to components whose behaviour can be changed dynamically in response to commands from external controllers.
}

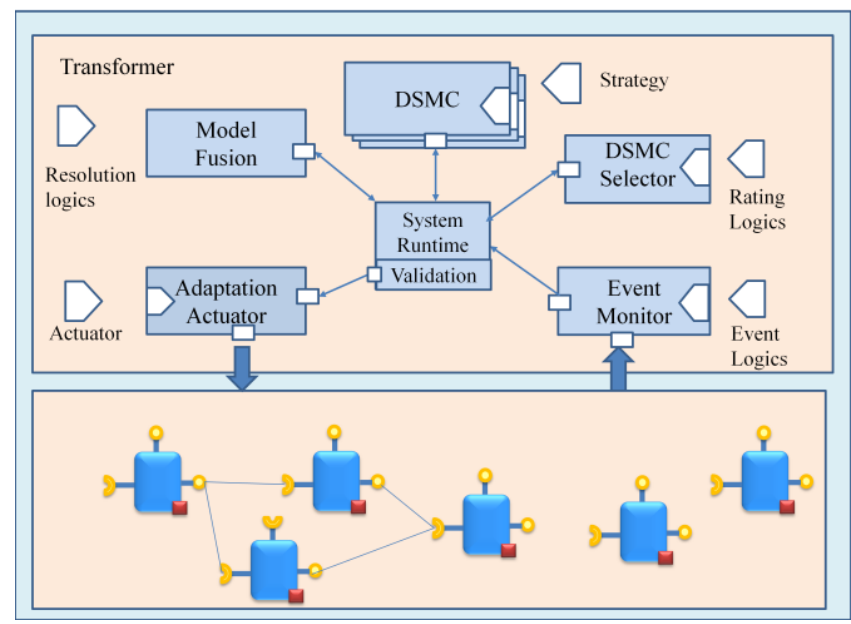

Figure 2. Transformer Framework Architecture

among these components. The SBR will automatically update the component configurations to keep the consistence between the adaptable component instances and their corresponding meta-objects.

- Event Monitor. The Event Monitor module observes and measures various system states. When certain condition is detected, it sends notifications to trigger a new round of the adaptation process. In current implementation, basic adaptation events, such as installing/updating/removing a component are natively supported.

- Adaptation Actuator. An Adaptation Actuator carries out the actual system modification by performing its corresponding adaptation actions. In current implementation, four basic types are natively supported: enabling components, disabling components, repairing a component, and changing a component's properties. These four actuators cover the two major classes of compositional adaptation methods: life-cycle management and parameter-based adaptation.

In Transformer, standard interfaces for Event Monitor and Adaptation Actuator are defined. Users can also define their custom Event Monitors and Adaptation Actuators by implementing those interfaces.

- Composable Adaptation Planner. A CAP encapsulates adaptation polices that control and coordinate adaptable applications constructed by adaptable components. Each CAP only contains a set of adaptation policies focusing on particular adaptation goal(s). CAP is introduced in Transformer to enhance adaptation modules' reusability and composability. This is in contrast with many frameworks in which adaptive controllers are typically coarse-grained (normally with one single unit) and interwoven with adaptation mechanisms. The next subsection will introduce CAP's design principles as well as corresponding component model.

- CAP Selector. As multiple CAPs are supported in Transformer and each provides a particular adaptation feature, not all these adaptation features are needed in every context. CAP Selector is introduced to select the appropriate set of CAPs according to current context 
adaptation requirements. By changing the set of employed CAPs according to the changing context, this module helps Transformer to support meta-adaptation.

- Model Fusion. As different CAPs encapsulate highly diverse adaptation policies and are developed individually, they might generate conflicting adaptation plans. The Model Fusion module aims at identifying and resolving such conflicts. By fusing those computed adaptation plans into a conflict-free one, this module effectively creates a global adaptation model with multiple adaptation concerns.

- Validation. Since the global adaptation model is constructed during run-time, it is possible that the fused adaptation model is not thoughtfully tested. Accordingly, the pluggable validation module is introduced to detect and prevent the system to take unintended adaptation actions. Third party plug-ins can use its provided service to change the fused adaptation plan if necessary. For instance, stop performing any adaptation action when the system repeatedly enters the same configuration within a user-specified time interval.

As this paper focuses on the adaptation composition and conflict resolution, in the following sections, detailed design of related modules - CAP, CAP Selector and Model Fusion - will be introduced. Information on SBR, Event Monitor and Adaptation Actuator can be found in our previous work [18].

\section{B. Reusable and Composable Adaptation Controller}

A CAP in Transformer contains adaptation logics for system managed adaptable components and is responsible for taking adaptation decisions that let those components adapt to changes in the environments.

According to Salehie's review [19], all 16 surveyed projects on self-adaptive systems, to a certain degree, separate adaptation logics from application business logics. This separation makes system adaptation logics easy to be revised. However, such separation alone does not guarantee adaptation modules to be reusable and composable. Additional design principles are needed to achieve adaptation composition.

\section{1) Design principle}

The following design principles are identified from our research experience to facilitate adaptation composition.

Orthogonality: This principle is meant to lower the chance of interference of concerns and reduce the complexity this interference may bring to the later conflict detection and resolution process. This is quite similar to the orthogonality design principle of Aspect Oriented Programming (AOP) [20]. However, due to the existence of the Model Fusion module, in Transformer, this feature is not mandatory required - though highly preferable.

Clear separation: This principle aims at enhancing the reusability of adaptation modules. We addressed this principle by forbidding a CAP to perform any adaptation action inside its reasoning logic. From this perspective, the CAPs are side-effect free - they can only expose their adaptation decisions to the Model Fusion through their predefined interface and have no direct impact towards system

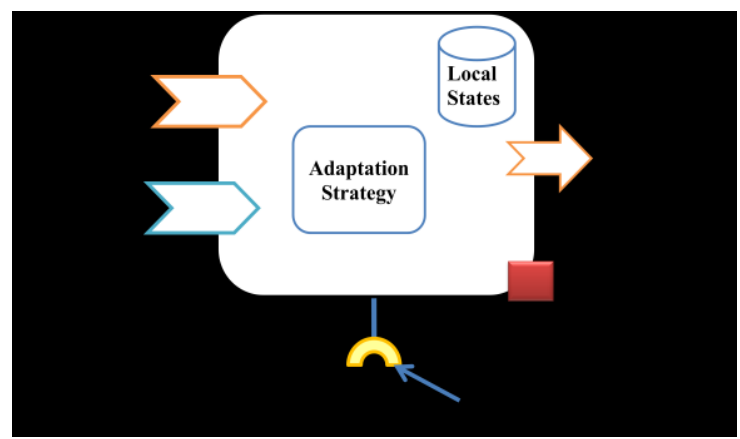

Figure 3. CAP Component Model

configurations. All adaptation plans will be sent to Model Fusion rather than being directly executed by CAPs. This design allows conflicts between different CAPs to be easily captured and resolved.

Restriction on adaptation actions: A CAP can only use adaptation actions with well-defined semantics. Relationships among all supported adaptation actions should also be clearly defined. This requirement is to promote composability and to simplify the conflict detection and resolution performed in the Model Fusion process.

A CAP is designed to be individually developable and deployable and can be used together with other CAPs. From this perspective, it is quite similar to aspects in AOP. However, in the AOP-based solutions, aspects may be attached virtually to any position of the original source code and adaptation logics mix with adaptation actions. In comparison, our work clearly defines what a CAP can do (that is, adaptation interface and action sets) as well as when it can do it (that is, only during adaptation process). Therefore conflicts identification and resolution can be largely simplified.

2) CAP Component

Following these principles, we developed the CAP component model. Each CAP must implement a predefined CAP interface to provide a general way of usage.

\section{a) Functional definition}

As we can see from Fig. 3, a CAP can be seen as a transducer which maps inputs - system information, events, and context - into a set of adaptation actions.

In order to make accurate adaptation, CAP needs accurate information for the current system configuration and context which is managed by SBR. When a CAP is invoked, a reference towards system global image will be provided by SBR. This reference allow CAP to get current snapshot of system status - for instance, a list of currently enabled components, a list of disabled components, a map of component dependences, etc. SBR also provides the reference of the context manager that maintains the latest context values. The change events are sent from Event Monitor. Detailed information about the functional interface can be found in [26].

In Transformer, an adaptation plan consists of a list of adaptation actions and their corresponding parameters. For instance, enabling component $\operatorname{com}_{\mathrm{a}}$ would be (enable, 
$\operatorname{com}_{\mathrm{a}}$. id). Here, we should point out that: according to our second design principle, a CAP only exposes its adaptation plan to Transformer rather than performing it via itself. Furthermore, only those actions supported by Transformer as specified in the Actuator Model - will be processed. Any other action will be ignored.

\section{b) Context dependences}

As CAPs are selected and employed during run-time, a context description mechanism is needed to describe a CAP's preferred applicable context. With this information, CAP Selector can calculate the matching degree between the CAP's preferred context and the system's current context. This information is represented in the meta-data attached with the CAP component based on our DRCom component model [11]. A simple constraints description format ua.mw.language.DCMD - was designed to describe the preferred context that a CAP is designed to work within.

Context preference: Each CAP's context preferences are written in the following format:

\section{Context Factor, Preferable Value, Impact Factor;}

separated by commas. Context Factor denotes the name of context factors that will influence the CAP's performance. Preferable Value denotes the preferred value of this context factor - normalized into range $[0,1]$. The Impact Factor is a normalized weight that expresses the influence of these context factors. If Impact Factor of a context factor $\mathrm{C}^{\mathrm{i}}$ is expressed as $w^{i}$, and the total number of related context factors is $\mathrm{N}$, then the following should hold:

$$
\sum_{\mathrm{i}=1}^{\mathrm{N}} w^{i}=1
$$

The preferable context of the CAP for self-healing (denoted as CAPrepair) can be expressed as follows:

option.selfhealing, 1, 0.8;

resource.cpuusage, $0,0.2$

It shows that this CAP's preferable value of option.selfhealing is 1 with impact factor 0.8 and resource.cpuusage is 0 with impact factor of 0.2 . In other words, it expresses the fact that this CAP best fits for the context with self-healing option set to 1 (true) and CPU usage is very low. This meta-data can be assigned by CAP developers and is easily reconfigured during deployment time.

\section{CAP Selector}

As shown in the motivational example, each CAP can only effectively conclude its adaptation actions when the current context matches its contextual requirements. In order to provide accurate adaptation behaviour, for a particular context, only a part of the installed CAPs should be used for the adaptation process. CAP Selector handles this selection process. Accordingly, one important responsibility of $C A P$ Selector is to calculate the similarity between each CAP's preferred context requirements and system's current context. We refer to this similarity as to the Context Matching Degree (CMD).

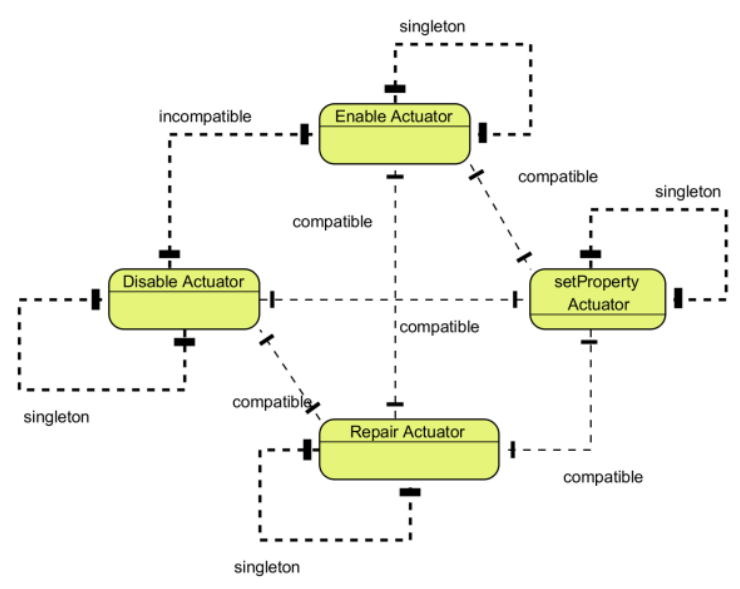

Figure 4. Actuator Model with Four Types of Actuators

In this paper, we use the inverse distance between system's current context and a CAP's preferred context to express such context similarity. As different context factors might normally have different levels of impacts towards the CAP's usability, the inverse distance is weighted by the impact factor. The context matching degree for any given $\mathrm{CAP}$, say $\mathrm{CAP}^{\mathrm{a}}$, is calculated by the following formula:

$\operatorname{CMD}\left(\mathrm{CAP}^{\mathrm{a}}\right)=\sum_{i=1}^{N} \frac{1}{1+K * A b s\left(C_{i}^{\text {current }}-C_{i}^{\text {opt }}\right)} * w^{i}$

in which the $C_{i}^{o p t}$ denotes the preferred value of the context factor $C^{i}$. The function Abs provides the absolute distance between $\mathrm{C}_{i}^{\text {opt }}$ and current value $C_{i}^{\text {current }}$ of $C^{i} . w^{i}$ is the impact factor for $\mathrm{CAP}^{\mathrm{a}}$. $\mathrm{K}$ is a constant that is used to adjust context sensitivity and is currently arbitrarily set to 9 . According to the calculated CMD, if the CMD of a CAP is higher than a certain threshold, this CAP will be selected. In this prototype, an arbitrary value -0.3 - is assigned.

As an example, if the current context is $\{\{$ option.selfhealing, 1$\},\{$ resource.cpuusage, 0.5$\}$, the CMD for CAPrepair described in the previous section - with preferred context $\{$ option.selfhealing, 1$\} \quad,\{$ resource. cpuusage, 0$\}\}$ - can be calculated with (2) as:

$\operatorname{CMD}\left(\mathrm{CAP}^{\text {repair }}\right)=0.8 /(1+9 * \mathrm{abs}(1-1))+0.2 /(1+9 *$ abs $(0.5-$ $0))=0.8363$

This value is bigger than the threshold (0.3), thus CAPrepair will be used for adaptation in this context.

One special case for the CAP Selector is that the CAP for software structural maintenance (denoted as $C A P^{\text {structure }}$ ) will be always chosen, as maintaining the integrity of the software structure is needed whatever the context. Of course, the function provided here is only one possible solution for CMD calculation. Other algorithms, such as the semantic graph matching schemes [21] and the context matching condition [15], can also be used in our framework.

\section{Model Fusion}

As described in Sect. III.A, the process of combining different CAPs and resolving possible conflicts becomes vital in guaranteeing system correctness. In this process, 
possible generated conflicts are identified according to the semantics of adaptation actions. For instance, the "enable" and "disable" actions cannot be performed towards a same component in one adaptation step. In order to describe semantics of adaptation actions and their relationships, the Actuator Model is provided.

\section{1) Actuator model}

Transformer defined a set of interfaces to specify the relationships between actuators - IActuatorModel for actuator model and IRelationship for the definition of relationships. Detailed description can be found in [26]. In current prototype, an Actuator model with four types of actuators is defined. In this model, three types of relationships are defined: compatible, incompatible and singleton. The relation "incompatible" between two actuators means these two adaptation actions cannot be performed towards the same adaptable object. Depending on the types of actuators, it can be either a component instance or a component property. For instance, "enable" is incompatible with "disable". This means that those two adaptation actions should not be performed towards the same component instance. The "compatible" is the opposite. Relation "singleton" specifies that this action cannot be performed more than once towards the same adaptable object. As the same adaptation actions normally should not be performed more than once in one adaptation step, in this actuator model, all actuators are singleton in themselves.

As we can see from Fig. 5, this model is defined only based on the type of adaptation actuators. It is clearly a simplified implementation. However, Transformer allows custom actuator models to be plugged to support more complex relationships.

Thanks to the explicitly defined Actuator Model, it is comparably straightforward to identify possible conflicts. Whenever selected CAPs propose two incompatible adaptation actions or violate the singleton relationship, conflicts arise. When this happens, the Model Fusion module $\operatorname{logs}$ the conflicts and inquiries the conflict resolution sub-module to resolve the conflicts.

\section{2) Fusion Logics}

The Actuator Model only depicts whether two adaptation actions can be performed together. However, dealing with those identified conflicts remains challenging. Some researches ignore all the adaptation actions that might generate conflicts [22]. Other solutions resolve conflicts by averaging the desired parameter settings from several adaptation controllers [10], or select actions with highest utility gain to the system [8]. None of these approaches use context information in their conflict resolution process. This limitation, it is argued, makes these approaches inappropriate in changing environments.

In Transformer, the fusion logic is designed making use of the following guidelines:

1) Respect component functional dependence. As a component can only be activated/executed when its functional dependences are satisfied, it is necessary to respect the decision made byCAPstructure to maintain the correctness of software architecture.
2) Assume that CAPs with higher CMDs can provide more accurate adaptation actions than the CAPs with lower CMD. The run-time calculated CMD can be used as a priority in resolving conflicts between different CAPs.

Following this principle, five conflict resolution rules are defined. The first two rules are designed based on the principle one and the remaining three ones are designed based on guideline two.

Rule 1: A component can be enabled only if CAP $P^{\text {structure }}$ denotes it can be enabled;

Rule 2: A component must be disabled if CAP structure denotes it should be disabled.

As can be seen from the Actuator Model, two of the three relations can generate conflicts: singleton and incompatible.

Rule 3: If a conflict arises among different types of actuators, perform the one proposed by the set of CAPs for which the sum of their CMDs is the greatest.

This rule is designed to solve conflicts between different types of actuators. It can be used to solve conflicts between "enable" and "disable" actions for component $\operatorname{com}_{a}$.For instance, when $C A P^{i}$ proposes to enable component $\mathrm{x}$ with $\mathrm{CMD}=0.5$ and $C A P^{j}$ proposes to disable it with $\mathrm{CMD}=0.4$, then it means that the "enable" action matches more with the current context and should be selected. Otherwise, the "disable" action will be performed.

It is worth remarking how Rule 3 does not apply to CAP $P^{\text {structure }}$ as it is already covered by Rule 1 and Rule 2 .

Rule 4: if multiple identical adaptation actions ("enable", "disable" or "repair") are proposed by CAPs towards the sample components, only one instance will be performed.

As we can see from the Actuator Model, actuators are defined to be singleton, which means they can be prescribed only once. The conflict resolution for "enable", "disable" or "repair" is quite straightforward: only one action is presented in the fused adaptation plan. Rule 4 describes this resolution logic.

Rule 5: if multiple "setProperty" adaptation actions are proposed, the final value is calculated by a conflict resolution policy (as described below).

In order to solve the singleton conflict for "setProperty" actuator, many solutions can be used. Here, we provide two variants for conflict resolution: "winner-gets-all" and "weighted-average". The former one chooses the value proposed by the CAP with highest CMD and the latter one calculate the fused value by averaging the property values weighted with CMD

1. Winner-gets-all

In this variant, a final property value is assigned with the value calculated by the CAP with the highest CMD (among the conflicting set).

2. Weighted-average

If multiple CAPs propose different values for the same property of a component, the final value is given by the average over all assigned values weighted by the corresponding CMDs. Let us denote $\mathcal{C}^{a}$ as the conflict set 
Table 1. Projects are compared in terms of the following Taxonomy Facets: "-” (feature not supported,) "E/I" (External/Internal), "S/G" (Specific/Generic solution), "SoC" (Separation of Concerns), "AC" (Adaptation composition)

\begin{tabular}{|c|c|c|c|c|c|c|c|}
\hline \multirow{2}{*}{ Compared projects } & \multicolumn{3}{|c|}{ Framework Realization } & \multicolumn{4}{|c|}{ Adaptation construction } \\
\hline & Target & $\mathrm{E} / \mathrm{I}$ & $\mathrm{S} / \mathrm{G}$ & SoC & $\mathrm{AC}$ & $\begin{array}{l}\text { Adaptation } \\
\text { Reuse }\end{array}$ & Meta-Adaptation \\
\hline Self-healing [1] & Components. & E & Specific & $\begin{array}{l}\text { Business -> } \\
\text { healing }\end{array}$ & - & - & - \\
\hline Rainbow[2] & Components & E & Generic & $\begin{array}{c}\text { Composable } \\
\text { adaptation }\end{array}$ & Utility function & $\begin{array}{c}\text { Yes, source code } \\
\text { level }\end{array}$ & - \\
\hline Gravity [4] & $\begin{array}{c}\text { Service } \\
\text { Components }\end{array}$ & E & Generic & $\begin{array}{c}\text { Business - } \\
>\text { Dynamicity }\end{array}$ & - & - & - \\
\hline Transformer & $\begin{array}{c}\text { Service } \\
\text { Components }\end{array}$ & E & Generic & $\begin{array}{c}\text { Composable } \\
\text { adaptation }\end{array}$ & $\begin{array}{l}\text { Actuator } \\
\text { semantic }\end{array}$ & Yes, components & Supported \\
\hline AAOP[6] & $\begin{array}{c}\text { Apps./ } \\
\text { Components }\end{array}$ & E & Generic & $\begin{array}{c}\text { Business - } \\
\text { >adaptation }\end{array}$ & - & Yes, aspects & $\begin{array}{c}\text { Select one adaptation } \\
\text { aspect }\end{array}$ \\
\hline $\begin{array}{c}\text { Opportunistic Integration } \\
{[7]}\end{array}$ & $\begin{array}{c}\text { Service } \\
\text { Components }\end{array}$ & E & Generic & $\begin{array}{c}\text { Composable } \\
\text { adaptation }\end{array}$ & Opportunistic & $\begin{array}{c}\text { Yes, } \\
\text { components }\end{array}$ & - \\
\hline
\end{tabular}

(the set of all CAPs proposing different values for the same property $a$ ); then the final value $a^{*}$ is calculated via (3) (where $a^{i}$ denotes the value assigned to property $a$ by $C A P^{i}$ ).

$\operatorname{prop}\left(a^{*}\right)=\frac{\sum_{i=1}^{\operatorname{size}\left(\mathcal{C}^{a}\right)} a^{i} * C M D_{C A P^{i}}}{\sum_{i=1}^{s i z e\left(\mathcal{C}^{a}\right)} C M D_{C A P^{i}}} C A P^{i} \in \mathcal{C}^{a}$

In current version, due to the limited model for actuators, the conflict resolution logics only focus on whether two adaptation actions can be performed together in the same step of adaptation. We are working to extend current model so as to allow conflict identification with other features such as two adaptation actions requiring to be always performed in a certain temporal order.

\section{CASE STUDY: ADAPTIVE VIDEO CONFERENCING SYSTEM WITH CHANGING CONCERNS}

This section describes our current implementation of Transformer through the conferencing system introduced in Sect. II.A.

\section{A. Overview}

A simplified version of the conferencing client shown in Fig. 1 is implemented through the DRCom model and used as our adaptation target. As shown in Fig. 1, it consists of three major subsystems: text messaging subsystem, local media capture subsystem and remote media player.

Equinox [23], an open source OSGi [24] implementation, is used as our basic development platform. The implementation of SBR and the CAP for structural maintenance are tailored from our previous work. For detailed implementation about these two modules, please refer to [18]. In this video conferencing system, functions for audio/video capturing, encoding and decoding are implemented by using Xuggle [25] - a java based package that wraps the FFMpeg toolset and provides rich support for video/audio processing. Those video processing modules are augmented with DRCom interface and meta-data so they can be managed by the Transformer framework. For instance, the property framerate of the Video Encoder component (used in local video capture subsystem) can be adapted during run-time.

In order to achieve the adaptation scenarios described in the motivational example, four CAPs are used: CAPstructure for software structure maintenance; 2) CAP ${ }^{\text {repair }}$ for SelfHealing 3) CAPquality for Quality Optimization 4) CAP ${ }^{\text {battery }}$ for Battery optimization.

CAP ${ }^{\text {structure }}$ is natively provided by the framework. The remaining three CAPs are domain-specific and are plugged into the system to enrich system adaptation capability. In order to execute properly, some CAPs might require additional monitors and actuators. For example, CAPrepair requires a custom Event Monitor to trigger a periodic examination for components' health and a custom Adaptation Actuator for component repairing.

\section{B. CAP adaptation logic}

In this case study, four different CAPs are involved in the adaptation process. A summary of key (simplified) adaptation logics is described in Fig. 5. As shown in this figure, $C A P^{\text {structure }}$ has two major logics: 1) if a component becomes "structurally satisfied" - that is, all its required interfaces have corresponding provided interfaces - then

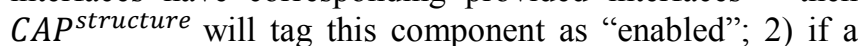
component becomes "structurally unsatisfied", this component will be tagged as "disabled". As the DRCom model is based on service-oriented architecture, dependences between components are binded/unbinded during run-time. This module provides basic supports for compositional adaptation and maintains the software architecture. Detailed discussion on this can be found in our previous paper [18].

As for the adaptation logics of CAPrepair, this component queries all enabled business components' properties triggered by a custom Event Monitor (Timer) and records these data in its local cache. The query action is achieved by using the management interface implemented by all DRCom components. When the component is not responsive to external queries (raises exceptions), we denote 


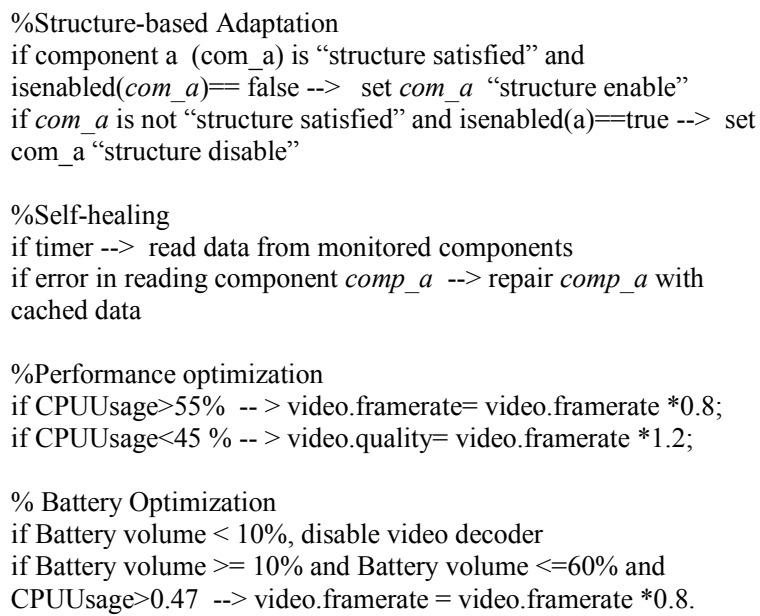

Figure 6.Example CAP Adaptation Logic

this component as "unhealthy" and in need of being repaired. This CAP will issue a "repair" adaptation action with the latest values of the cached properties. The "repair" action is the only action it will issue in its adaptation plan.

CAP quality adjusts video quality according to system available resources. When there is enough CPU (less than $45 \%$ ), it increases video quality. When the CPU usage is too high (greater than 55\%), this adaptation module decreases video quality so as to save some resource for other applications. The encoder's quality is adjusted by setting the encoding frame rate. Here, the frame rate is chosen as the adaptation target as it has an obvious impact towards application CPU usage and video quality. Similarly, the adaptation logics of $C A P^{\text {battery }}$ adapt encoder's performance according to current state of battery. When the battery is below certain low threshold $(10 \%)$, it will disable the encoder component. If the remaining battery is within an acceptable range $-[0.1,0.6]$ - but the rate of battery usage is too high (greater than $47 \%$ ), it will reduce the quality of encoding by reducing the frame rate of encoded video.

In this case study, we keep these adaptation logics simple and focus only on conflict detection and resolution. These simple logics can already generate several conflicting scenarios. For instance, the $C A P^{\text {battery }}$ will try to reduce framerate while $C A P^{\text {quality }}$ will take the opposite path trying to enhance the framerate whenever CPU allows.

\section{EvaluATIONS}

We evaluate Transformer from different perspectives. Firstly, a qualitative analysis is provided.

\section{A. Qualitative Analysis}

We compare Transformer with five other different adaptation frameworks/solutions from two major perspectives: framework realization and adaptation concerns.

As can be seen from Table 1, except for the AAOP project that uses aspects to achieve adaptation, the target for adaptation is focused on the component level. All the considered projects use external adaptation loops that isolate adaptation logics from the application business logic. Table 1 also shows that most solutions are generic and can be used for different adaptation requirements. These similarities show that these design choices have been adopted by most approaches and are becoming a general practice.

However, in terms of the problem of how to build adaptation behaviour, much less agreement has been reached among researchers. In terms of separation of concerns, different levels of separation are being used. Many approaches (Self-healing, AAOP, Gravity) do not support the adaptation composition. Except for Transformer, only Rainbow provides explicit conflict resolution support with utility-based solution. As for the meta-adaptation, only AAOP provides limited support; however, it only allows one adaptation aspect to be used at any given time.

For the adaptation reuse, in Transformer, programmers can directly reuse adaptation modules in binary form. This feature makes the implementation of adaptation logics much simpler. Although other two approaches (AAOP and "Opportunistic Integration") also provide certain level of adaptation reusability, their adaptation modules normally are not designed for composition. Rainbow does provide a certain level of adaptation reuse. However, in Rainbow, adaptation logics have to be written in a custom, nonstandard language ("Stich"). Binary reuse of adaptation modules cannot be achieved. The dependence to a custom language also brings additional complexity to the end users.

Transformer, as shown in Table 1, provides a general adaptation framework that can be used for different adaptation logics. It allows different adaptation logics to be reused and composed into more complex adaptation modules and reused in different contexts. It supports metaadaptation by means of the CAP Selector and allows conflicts to be systematically identified and resolved by providing its Actuator Model. For detailed comparisons with other projects and from other angles please refer to [26].

\section{B. Multiple CAP performance evaluation}

In this section, we test the overhead introduced by composing multiple adaptation logics with respect to the standalone approach. Each simulation is performed 1000 times and the average values are used to soften the impact

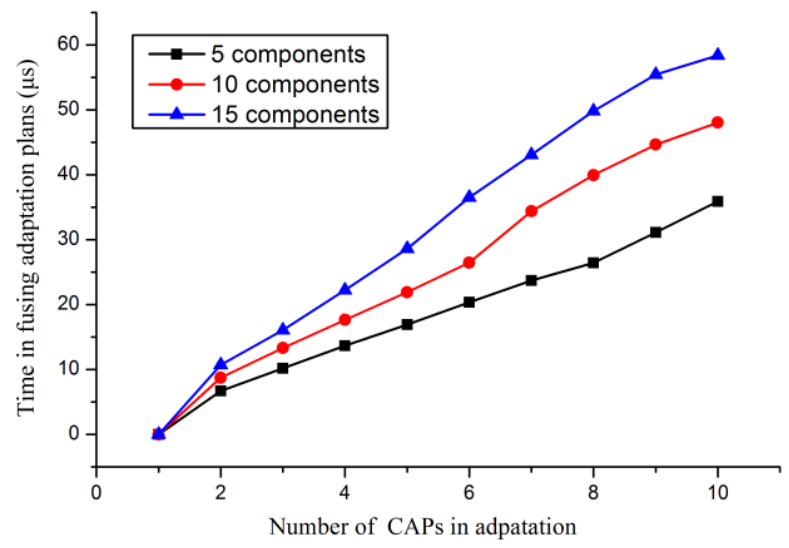

Figure 5. Model Fusion Performance 
introduced by e.g. Java's garbage collection. As hardware platform we used a DELL Latitude D630 laptop with Intel T7200 CPU. The JVM we adopted is Sun JAVA 1.6.2 SDK on Windows.

Model Fusion Time: One key overhead introduced in our approach is due to the Model Fusion process. In order to analyse the performance of such process, we introduced one simple CAP, which only iterates through all installed components and gets their properties. This CAP will "enable" all installed components and execute the same "setProperty" action for each component. This CAP is deployed multiple times (ranging from 1 to 9) to simulate multiple CAP adaptation. Each CAP instance computes actually exactly the same adaptation plan. By doing so, we introduce a number of conflicts in violating the singleton relationship.

At this point, the plan generated by different CAPs will be fused into one adaptation plan according to the fusion rule set with "weighted-average". We can see that the Model Fusion time depends on two basic factors: one is the number of managed components -that determines the issued "enable" and "setProperty" actions - and the other factor is the number of utilized CAPs. As shown in Fig. 6, the more CAPs are used, the more Model Fusion time is required. However, according to our programming practices, for any given context, normally less than 5 CAPs will be used. This means that for each round of adaptation, less than $30 \mu \mathrm{s}$ fusion time can be expected.

\section{Case study-based Model Fusion analysis}

This section demonstrates how different model fusion implementations can influence the system final adaptation behaviour. The target application is the Video conferencing system described in Sect. II. In order to calculate CMDs, a simple context manager is used to provide current context values for CPU and remaining battery. As it is hard to accurately retrieve the remaining battery level, we simulated its consumption rate through (4). Such formula calculates the consumption rate by using current frame rate $\mathrm{f}$ and a fixed number $\mathrm{N}$ representing the energy consumed by other application components as well as by the OS. The full battery is expressed as 1 . Rate $a$ is set to $8.333 \mathrm{e}-4$. This value is chosen to bring about 100 minutes of simulation time when both variable $f$ and $\mathrm{N}$ are set to 6 .

battery consumption rate $=a *(f+N) /$ minute

For the two different variants of Model Fusion logics, the only difference is the way they deal with the conflicts from property settings. As $C A P^{\text {repair }}$ and $C A P^{\text {structural }}$ will not use "setProperty" action, here, only CAPbattery and CAP quality will be discussed. In Fig. 7, the two different variants of fusing rule sets are compared to demonstrate their impact towards system performance. To empathize the effect of the conflict resolution process, in this simulation the threshold for CAP selection is set to 0 (all CAPs are selected). In order to make a clear comparison between adaptations with and without adaptation composition, adaptation processes making use of only one of these two CAPs are also shown in the same figure.

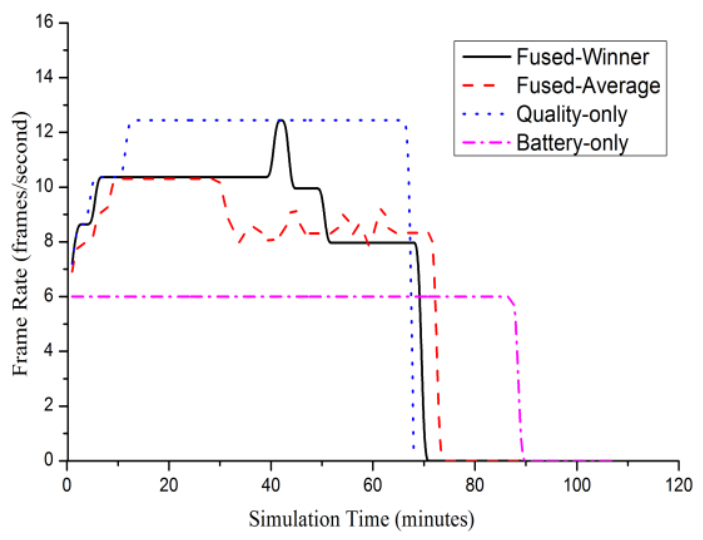

Figure 7 Adaptation with two different Model Fusion Policies

Among the three major subsystems, the "local video capture" and the "remote video player" have major impact towards the system performance as they both contain CPU intensive tasks. As we want to check the impact of adaptation for the local video capture subsystem, the remote video player subsystem decodes a uniform video (a video with slow-motion video frames, size $320 \times 220$ ). So the video decoder almost uses constant CPU to decode different parts of this video stream. The video-capture subsystem captures screen (with resolution 1280 times 800) with slight changing contents (practically, the debug output of the conferencing system). These settings, to some extent, factor out variations in the source of video from variations due to adaptation actions. The hardware is the same as the previous simulation. A custom designed Event Monitor triggers the adaptation every minute.

The initial setting of video encoder frame rate is 6 frame/sec. If only $C A P^{\text {quality }}$ is used, the frame rate will gradually increase to 12.44 frame/sec until the CPU usage is above $55 \%$. The high frame rate enhances the quality of the video conferencing system, however, at the cost of higher battery consumption. Fig. 8 shows that with $C A P^{\text {quality }}$ only, this application can only run 69 minutes with a full charged battery. However, when only CAPbattery is used, as it only adjusts the frame rate when battery is below 0.6 , the frame rate keeps low even when the CPU still has a lot of free computing power. Although this can achieve much longer battery life (about 111 minutes), it results in poor video quality in terms of frame rate. This shows that using only one of the CAPs cannot achieve a balance of quality and battery usage.

In comparison, when two CAPs are used together the final adaptation plan is constructed according to CAPs' CMDs. As shown in Fig. 9, in the "winner-gets-all" variant, the system adaptation behaviour can be classified into two different regions. When the $\mathrm{CMD}$ of $C A P^{\text {quality }}$ is bigger than the CMD of $C A P^{\text {battery }}$, system will try to optimize quality. Otherwise, the frame rate proposed by $C A P^{\text {battery }}$ will be used. This fast switch of adaptation model can partially explain the steep decrease of frame rate in time interval $[44,50]$ minutes. In contrast, the "weightedaverage" variant results in a smoother policy switching, as the frame rate largely keeps between 8 and 10fps. This 
policy keeps the system from sudden shifts and oscillations by gradually considering both adaptation concerns. These two variants both disable the encoding model when battery is below 0.1, to achieve more execution time. The "weighted-average" achieve around 91 minute execution time and for "winner-gets-all", it is around 92 minutes.

This simulation also shows that an adaptation action can also influence CAPs' CMDs that in turn will determine which adaptation actions shall be chosen. For instance, the CPU usage will be influenced by the adaptation actions taken by the CAP ${ }^{\text {quality }}$ and $C A P^{\text {battery }}$. Although it is not evident in this scenario due to the monolithic decreasing battery level, in other systems, it is possible to create a "ping-pong" effect that introduces adaptation loops and leads the system to an unstable state. That is this reason we introduced the Validation module in Transformer.

\section{CONCLUSIONS}

In this paper we have described our approach to support meta-adaptation with reusable adaptation modules. We have shown in particular how Transformer allows adaptation to be contextually constructed by composing reusable adaptation components. A service-oriented implementation has been shown to demonstrate the feasibility of our approach. As our framework makes strict definition of adaptation actions and adaptation interfaces, we are able to detect conflicts in a systematic way. Compared to other frameworks, our system can greatly enhance the reusability of adaptation components across multiple contexts. This approach has been compared with other adaptation frameworks from different perspectives. In addition to the system described in this paper, it has been successfully used in an autonomous robot control platform [27].

\section{ACKNOWLEDGMENT}

This work is partially supported by the Zhejiang Provincial Natural Science foundation of China under Grant No, LY12F02041 and Zhejiang Sci. \& Tech. Dept. Grant No. 2011C33G2060108 and by IBBT (the Interdisciplinary Institute for Broadband Technology), an independent research institute founded by the Flemish government.

\section{REFERENCE}

[1] S. Sicard, F. Boyer, et al., "Using Components for ArchitectureBased Management," Proc. 30th International Conference on Software Engineering (ICSE 08), 2008, pp. 101-110.

[2] D. Garlan, S. W. Cheng, et al., "Rainbow: Architecture-based selfadaptation with reusable infrastructure," Computer, vol. 37, pp. 4649, 2004.

[3] P. Oreizy, M. M. Gorlick, et al., "An architecture-based approach to self-adaptive software," IEEE Intelligent Systems \& Their Applications, vol. 14, pp. 54-62, May-Jun 1999.

[4] H. Cervantes and R. S. Hall, "Autonomous adaptation to dynamic availability using a service-oriented component model," Proc. 26th International Conference on Software Engineering (ICSE 04), 2004, pp. 614-623.

[5] J. Hillman and I. Warren, "Meta-Adaptation in Autonomic Systems," Proc. FTDCS, 2004, pp. 292-298.
[6] A. Janik and K. Zielinski, "Adaptability mechanisms for autonomic system implementation with AAOP," Software: Practice and Experience, vol. 40, pp. 209-223, 2010.

[7] Y. Maurel, A. Diaconescu, and P. Lalanda, "Creating Complex, Adaptable Management Strategies via the Opportunistic Integration of Decentralised Management Resources," Proc. International Conference on Adaptive and Intelligent Systems(ICAI 09), 2009, pp. 86-91.

[8] S. Cheng, "Rainbow: Cost-effective, Software Architecture-based Self-adaptation," PhD Thesis, CMU, 2008.

[9] L. Capra, W. Emmerich, and C. Mascolo, "CARISMA: Contextaware reflective mlddleware system for mobile applications," IEEE Transactions on Software Engineering, vol. 29, pp. 929-945, 2003.

[10] G. B. Patrick, "Cholla: A Framework for Composing and Coordinating Adaptations in Networked Systems," IEEE Transactions on Computers, vol. 58, pp. 1456-1469, 2009.

[11] N. Gui, V. De Florio, et al., "A framework for adaptive real-time applications: the declarative real-time OSGi component model," Proc. 7th Workshop on Adaptive and Reflective Middleware(ARM 08), 2008, pp. 1-6.

[12] P. K. McKinley, S. M. Sadjadi, et al., "Composing adaptive software," Computer, vol. 37, pp. 56-64, Jul 2004.

[13] V. De Florio, "A Fault-Tolerance Linguistic Structure for Distributed Applications," PhD Thesis, Dept. of Electrical Engineering, Katholieke Universiteit Leuven, 2000.

[14] J. Dowling and V. Cahill, "Self-managed decentralised systems using K-components and collaborative reinforcement learning," Proc. 1st ACM SIGSOFT workshop on Self-managed systems, 2004, pp. 3943.

[15] K. Fujii and T. Suda, "Semantics-based Context-aware Dynamic Service Composition," ACM Transactions on Autonomous and Adaptive Systems, vol. 4, pp. 12-42, May 2009.

[16] E. P. Kasten and P. K. McKinley, "Perimorph: Run-time composition and state management for adaptive systems," Proc. 24th International Conference on Distributed Computing Systems Workshops, 2004, pp. 332-337.

[17] B. H. C. Cheng, R. de Lemos, H. Giese, et al., "Software Engineering for Self-Adaptive Systems: A Research Roadmap," Software Engineering for Self-Adaptive Systems, vol. 5525, pp. 1-26, 2009.

[18] N. Gui, V. De Florio, et al., "Toward architecture-based contextaware deployment and adaptation," Journal of Systems and Software, vol. 84, pp. 185-197, 2011.

[19] M. Salehie and L. Tahvildari, "Self-Adaptive Software: Landscape and Research Challenges," ACM Transactions on Autonomous and Adaptive Systems, vol. 4, pp. 14-55, May 2009.

[20] G. Kiczales, J. Lamping, et al., "Aspect-oriented programming," Ecoop'97: Object-Oriented Programming, vol. 1241, pp. 220-242, 1997.

[21] J. Kwon, O.-H. Choi, et al., "Deriving similarity for Semantic Web using similarity graph," Journal of Intelligent. Information System, vol. 26, pp. 149-166, 2006

[22] D. Preuveneers and Y. Berbers, "Towards Context-Aware and Resource-Driven Self-Adaptation for Mobile Handheld Applications," Applied Computing 2007, vol 1, pp. 1165-1170, 2007.

[23] Equinox - OSGi implementation, Available at: http://www.eclipse.org/equinox/

[24] Open Service Gateway Initiative, v4.1, http://www.osgi.org, 2010.

[25] Xuggle -. Available: http://www.xuggle.com

[26] N. Gui, "Middleware-based adaptation evolution with reusable adaptation components," PhD thesis, Unversity of Antwerp, 2012.

[27] N. Gui, P.J. Pientens, et al., "Run-time Reconfiguration Software Platform for Autonomous Robot," Proc of ADAMUS 2010, held in the 7th ACM International Conference on Pervasive Services, 2010, pp. 19-24. 